\title{
Effect of glucosamine on intraocular pressure
}

Hamed Esfandiari, ${ }^{1}$ Syeda Sumara Taranum Basith, ${ }^{2}$ Nils A. Loewen ${ }^{1}$

1-Department of Ophthalmology, School of Medicine, University of Pittsburgh, Pittsburgh, Pennsylvania, United States

2-Department of Ophthalmology, Northwestern University, Chicago, Illinois, United states

Corresponding author: Hamed Esfandiari, MD

E-mail: hamed.esfandiari@northwestern.edu 


\section{Abstract}

Glucosamine is the most common nutrition supplement used in the United States. It is promoted not only as a pain reliever but also a therapy to slow osteoarthritis. Although studies with 3,800 patients with osteoarthritis of the knee or hip found that glucosamine was no better than placebo, it continues to be used for these two indications due to its perceived safety. However, reports of an increased intraocular pressure are alarming, especially in glaucoma. Glycosaminoglycans play a key role in the physiology of the cornea, conventional outflow of aqueous humor and the retina. In theory, long-term treatment with glucosamine could reach a concentration that impacts the morphology and function of ocular tissues. We recommend ophthalmologists counsel glaucoma patients about the potential risks of glucosamine and encourage cessation in uncontrolled intraocular pressure.

Keywords: Glucosamine, intraocular pressure, glaucoma, osteoarthritis, medication side effects. 


\section{Introduction}

Nutritional supplements are intended to provide nutrients that may otherwise not be consumed in sufficient quantities in a regular diet, including vitamins, minerals, proteins, amino acids or substances. Their use has increased since the 1970 s and is now reported by $49 \%$ of the US population. Glucosamine is the most commonly prescribed supplement for $\mathrm{OA}[1]$ as an attempt to slow disease progression.[2,3] Glycosaminoglycans (GAGs) are the main constituent of the cartilage and are also abundant in corneal stroma and contribute morphological and functional characteristics to the trabecular meshwork of conventional outflow pathway.[4,5]

While the outflow capacity of the TM is partially regulated by the content of GAGs in the trabecular meshwork and could be directly affected by exogenous GAGs, [6] applanation tonometry measurement is also affected by GAG-induced changes in the biomechanical properties of the cornea.[5,7]

Since both glaucoma and OA are age-related morbidities, any systemic glucosamine consumption for osteoarthritis has potential to also affect glaucoma in the same individual.[8]

\section{Glycosaminoglycans}

Glycosaminoglycans (GAGs) are a family of highly sulfated, complex, polydisperse linear polysaccharides that display various important biological roles.[9] GAGs are categorized based on the difference of repeating disaccharide units; the most common GAG structures are chondroitin sulfate, dermatan sulfate, heparan sulfate, keratan sulfate, hyaluronic acid, and heparin.[9]

GAGs are integral components of basement membranes and interact with many extracellular matrix (ECM) components, including laminin, collagen, and fibronectin and are essential for cell adhesion and migration.[10] This interaction determines the architecture and permeability of the basement membrane.[11] Cartilage in the joint is mainly composed of type II collagen and glycosaminoglycans. Negatively charged GAGs absorb water and sodium and contribute to the viscosity and resistance to compression and shear stress of cartilage.[12]

Glucosamine is an amino monosaccharide and an essential constituent of chondroitin and keratin sulfate, a principal GAG in cartilage. It was first thought that glucosamine works by providing building blocks for the repair of the ECM of cartilage. However, anti-inflammatory and anti-catabolic effects have also been attributed to it.[13] 
Despite these roles, the effectiveness of glucosamine to treat OA is debated. A recent meta-analysis of ten randomized clinical trials with a total sample size of 3803 patients found no significant differences in joint pain reduction or joint space narrowing between placebo and glucosamine.[14]

The Glucosamine/Chondroitin Sulfate (CS) Arthritis Intervention Trial (GAIT) - the largest clinical trial examining the efficacy of glucosamine and CS in OA compared celecoxib, glucosamine, CS, the combination of glucosamine and CS, and placebo in a multi-center double-blind randomized study.[15] A total of 1583 individuals with symptomatic OA were randomly assigned to receive daily doses of $1500 \mathrm{mg}$ of glucosamine, $1200 \mathrm{mg}$ of CS, both glucosamine and CS, $200 \mathrm{mg}$ of celecoxib, or placebo for 24 weeks. The primary outcome measure was an at least $20 \%$ reduction in pain from baseline enrollment, measured using the Western Ontario and McMaster Universities Osteoarthritis Index (WOMAC) pain subscale. OA patient groups were stratified by severity of knee pain at baseline into mild, moderate and severe. Glucosamine, $\mathrm{CS}$, or their combination therapies were not significantly different from placebo in pain reduction $(p>0.05)$. Among the moderate to severe pain stratum group of patients, however, the combination of glucosamine and CS was significantly better than placebo in treating OA.

Despite such limited evidence glucosamine remains a popular treatment, partly due to its reputation for safety. Almost one in five Americans with OA takes glucosamine and the sales approached \$730 in 2004.[16]

\section{Glycosaminoglycans and the eye}

GAGs are the most abundant heteropolysaccharides in the human eye.[17] They are a key constituent of the extracellular matrix and act as a filler substance between cells and fibers in tissues. Here, they bind to proteoglycans, matrix proteins, and hyaluronan and form larger complexes.

The normal aqueous outflow of the eye is affected by the content of GAGs, in particular in the juxtacanalicular part of the trabecular meshwork[18,19] in a process termed funneling.[20] The trabecular meshworks of primates contain significant amounts of the major glycosaminoglycan found in the ECM of other tissues. [4] Side chains of glycosaminoglycans interact to form a system of entangled chains of polyanionic macromolecules, hence, act like a gel and are an important contributor to outflow resistance.[5]

It has been shown that high level of tumor growth factor beta 2 (TGFb2) in glaucomatous aqueous stimulates trabecular cells to secrete a high level of GAGs, which cross-links proteins to complexes not degradable by metalloproteinases.[21] Histologic evaluation of trabecular meshwork in steroid-induced 
glaucoma shows GAG in the TM. Although myocilin is the most characteristic ECM component for glucocorticoid-induced glaucoma,[22] additional ECM and constriction of the trabecular spaces can be observed in sensitive subjects even after a short course.[23]

Exogenous GAG is also shown to stimulate cultured human trabecular meshwork cells to produce more GAG as a positive feedback mechanism.[24]

\section{Clinical studies on the effect of glucosamine on IOP}

In a small retrospective study, Murphy et al. noted oral glucosamine supplement was associated with a significant but reversible rise in pressure.[8] They observed that poorly controlled glaucoma patients had controlled IOP once glucosamine supplementation was discontinued, and IOP increased in those patients with glaucoma who received glucosamine supplement for their OA condition. Shortcomings acknowledged by the authors included variability in glucosamine dosage and length of treatment, compliance, and brand variability. Furthermore, as GAG are also abundant in the corneal stroma,[25] any change in its concentration could lead to IOP measurements error as applanation tonometry is influenced by biomechanics of the cornea. While this study found an association between taking glucosamine and increased eye pressure, it did not establish a cause-and-effect relationship.

To address the shortcomings of mentioned study, we conducted a randomized clinical trial to explore the possible role of glucosamine supplement in the rise of IOP in patients with OA.[26] Eighty-eight patients with osteoarthritis who attended a rheumatology clinic were included. Patients with eye diseases that might affect the biomechanics of the cornea, including a history of ocular surgery, corneal scar, and dystrophies were excluded. Forty-four patients were randomly assigned to take $750 \mathrm{mg}$ glucosamine three times a day for 3 months and 44 patients to take gelatinous capsules filled with sugar as a placebo on the same schedule.

The rise in the IOP was significantly larger at 3-month follow-up visit in the glucosamine group. In the glucosamine group, $34 \%$ of patients had an IOP increase of more than $2 \mathrm{~mm} \mathrm{HG}$ compared to $23.5 \%$ of patients in the placebo group. Additionally, the mean age in those with increases of $2 \mathrm{~mm} \mathrm{Hg} I O P$ or more was 66 years, compared to 57.7 years in patients who had increases of less than $2 \mathrm{~mm} \mathrm{Hg}$. Accumulation of extracellular matrix and thickening of the basement membrane could be aggravated by the exogenous use of GAG and compromise the reduced function of the trabecular meshwork. This is supported by histology that demonstrated age-related structural and functional changes including loss of cellularity, collagen and 
extracellular matrix accumulation, thickening, and fusion of trabecular sheets.[6,27] Age is also shown to be a risk factor for steroid-induced ocular hypertension.[23] The risk of ocular hypertension was not associated with diabetes mellitus, cardiovascular disease or gender. The results of our study showed that while glucosamine causes a statistically significant increase in IOP in patients with osteoarthritis, corneal biomechanics remain unchanged within 3 months of glucosamine supplement therapy. These results suggest that glucosamine supplementation could cause a trabecular meshwork selective change after 3 months.

There is no study concerning the pharmacokinetics of glucosamine in the trabecular meshwork. In OA, the glucosamine concentration in synovial fluid after oral administration is 500 -fold less than the level expected to have a therapeutic effect on joint space narrowing[28] but it is possible that oral supplement reaches problematic levels in the TM instead. Another theory to explain why IOP might be more affected than joint parameters in $\mathrm{OA}$ is that high concentrations of GAG in aqueous could draw more water into the anterior chamber due to an osmotic effect, thus inducing swelling and pore size compromise, leading to a reduced outflow.[5]

\section{Conclusions}

Although the effect of Glucosamine on osteoarthritis is debated and recent studies indicate a placebo effect, it is unlikely that patients will discontinue as any alleviation is a benefit. However, the recent clinical association between glucosamine use and an IOP elevation is concerning and matches older laboratory studies. We recommend ophthalmologists share this information with their glaucoma patients and alert them to the potential dangers of nutritional supplements that are not regulated by the FDA.

Glycosaminoglycans should be discontinued in glaucoma patients with an uncontrolled IOP. 


\section{Reference}

1. Deal CL, Moskowitz RW. Nutraceuticals as therapeutic agents in osteoarthritis. The role of glucosamine, chondroitin sulfate, and collagen hydrolysate. Rheum Dis Clin North Am. 1999;25: 379395.

2. Bucsi L, Poór G. Efficacy and tolerability of oral chondroitin sulfate as a symptomatic slow-acting drug for osteoarthritis (SYSADOA) in the treatment of knee osteoarthritis. Osteoarthritis Cartilage. 1998;6 Suppl A: 31-36.

3. Richy F, Bruyere O, Ethgen O, Cucherat M, Henrotin Y, Reginster J-Y. Structural and symptomatic efficacy of glucosamine and chondroitin in knee osteoarthritis: a comprehensive meta-analysis. Arch Intern Med. 2003;163: 1514-1522.

4. Acott TS, Westcott M, Passo MS, Van Buskirk EM. Trabecular meshwork glycosaminoglycans in human and cynomolgus monkey eye. Invest Ophthalmol Vis Sci. 1985;26: 1320-1329.

5. Knepper PA, McLone DG. Glycosaminoglycans and outflow pathways of the eye and brain. Pediatr Neurosci. 1985;12: 240-251.

6. Vranka JA, Kelley MJ, Acott TS, Keller KE. Extracellular matrix in the trabecular meshwork: intraocular pressure regulation and dysregulation in glaucoma. Exp Eye Res. 2015;133: 112-125.

7. Luce DA. Determining in vivo biomechanical properties of the cornea with an ocular response analyzer. J Cataract Refract Surg. 2005;31: 156-162.

8. Murphy RK, Ketzler L, Rice RDE, Johnson SM, Doss MS, Jaccoma EH. Oral glucosamine supplements as a possible ocular hypertensive agent. JAMA Ophthalmol. 2013;131: 955-957.

9. Zhang F, Zhang Z, Linhardt RJ. Chapter 3 - Glycosaminoglycans. In: Cummings RD, Pierce JM, editors. Handbook of Glycomics. San Diego: Academic Press; 2010. pp. 59-80.

10. Bartolmäs T, Heyn T, Mickeleit M, Fischer A, Reutter W, Danker K. Glucosamineglycerophospholipids that activate cell-matrix adhesion and migration. J Med Chem. 2005;48: 67506755.

11. Kefalides NA. Basement membranes: structural and biosynthetic considerations. J Invest Dermatol. 1975;65: 85-92.

12. Bhosale AM, Richardson JB. Articular cartilage: structure, injuries and review of management. Br Med Bull. 2008;87: 77-95.

13. Henrotin Y, Mobasheri A, Marty M. Is there any scientific evidence for the use of glucosamine in the management of human osteoarthritis? Arthritis Res Ther. 2012;14: 201.

14. Wandel S, Jüni P, Tendal B, Nüesch E, Villiger PM, Welton NJ, et al. Effects of glucosamine, chondroitin, or placebo in patients with osteoarthritis of hip or knee: network meta-analysis. BMJ. 2010;341: c4675.

15. Clegg DO, Reda DJ, Harris CL, Klein MA, O’Dell JR, Hooper MM, et al. Glucosamine, 
chondroitin sulfate, and the two in combination for painful knee osteoarthritis. N Engl J Med. 2006;354: 795-808.

16. Annual nutrition industry overview. Nutr Bus J. 2005; 6-7.

17. Pacella E, Pacella F, De Paolis G, Parisella FR, Turchetti P, Anello G, et al. Glycosaminoglycans in the human cornea: age-related changes. Ophthalmol Eye Dis. 2015;7: 1-5.

18. Rohen JW, Futa R, Lütjen-Drecoll E. The fine structure of the cribriform meshwork in normal and glaucomatous eyes as seen in tangential sections. Invest Ophthalmol Vis Sci. 1981;21: 574-585.

19. Rohen JW, Schachtschabel DO, Wehrmann R. Structural changes of human and monkey trabecular meshwork following in vitro cultivation. Graefes Arch Clin Exp Ophthalmol. 1982;218: 225232.

20. Overby DR, Stamer WD, Johnson M. The changing paradigm of outflow resistance generation: towards synergistic models of the JCT and inner wall endothelium. Exp Eye Res. 2009;88: 656-670.

21. Welge-Lüßen U, May CA, Eichhorn M, Bloemendal H, Lütjen-Drecoll E. aB-Crystallin in the Trabecular Meshwork Is Inducible by Transforming Growth Factor- $\beta$. Invest Ophthalmol Vis Sci. The Association for Research in Vision and Ophthalmology; 1999;40: 2235-2241.

22. Kersey JP, Broadway DC. Corticosteroid-induced glaucoma: a review of the literature. Eye . 2006;20: 407-416.

23. Jones R 3rd, Rhee DJ. Corticosteroid-induced ocular hypertension and glaucoma: a brief review and update of the literature. Curr Opin Ophthalmol. 2006;17: 163-167.

24. Binninger EA, Schachtschabel DO, Rohen JW. Exogenous glycosaminoglycans stimulate hyaluronic acid synthesis by cultured human trabecular-meshwork cells. Exp Eye Res. 1987;45: 169177.

25. Meek KM, Leonard DW. Ultrastructure of the corneal stroma: a comparative study. Biophys J. 1993;64: 273-280.

26. Esfandiari H, Pakravan M, Zakeri Z, Ziaie S, Pakravan P, Ownagh V. Effect of glucosamine on intraocular pressure: a randomized clinical trial. Eye . 2017;31: 389-394.

27. Becker B. The decline in aqueous secretion and outflow facility with age. Am J Ophthalmol. 1958;46: 731-736.

28. Laverty S, Sandy JD, Celeste C, Vachon P, Marier J-F, Plaas AHK. Synovial fluid levels and serum pharmacokinetics in a large animal model following treatment with oral glucosamine at clinically relevant doses. Arthritis Rheum. 2005;52: 181-191. 